\title{
EFECTO DE LA CÁSCARA O PULPA DE Solanum tuberosum SOBRE EL PESO DE ÓRGANOS Y ESTRÉS OXIDATIVO HEPÁTICO EN RATAS OBESAS
}

\author{
Viviana J. Ortiz-Rojas ${ }^{1, a}$, Nataly D. Bernuy-Osorio ${ }^{1, b}$, Otto A. Zea-Mendoza ${ }^{1, c}$, Carlos Vílchez-Perales ${ }^{1, d}$
}

\begin{abstract}
RESUMEN
Con el objetivo de determinar el efecto del consumo de harina de cáscara o harina de pulpa de dos variedades de Solanum tuberosum (papa Yungay y papa Canchán) sobre la acumulación de tejido adiposo, peso de órganos y estrés oxidativo en hígado de ratas realizamos un estudio experimental en 24 ratas Holtzman obesas, divididos en cuatro grupos y, sometidas a dietas que contenían $10 \%$ de harina de las dos variedades de papa. Los grupos fueron T1: cáscara Yungay, T2: pulpa Yungay; T3: cáscara Canchán; y T4: pulpa Canchán. Al finalizar, se sacrificaron todos los animales para registrar los pesos de órganos y tejido adiposo, y extraer muestras para determinar la actividad enzimática de superóxido dismutasa y catalasa en el hígado. El grupo de ratas obesas que consumió pulpa de variedad Yungay tuvo menor estrés oxidativo en el hígado; además, independientemente de la parte de tubérculo consumido, esta variedad redujo el peso de los riñones.
\end{abstract}

Palabras clave: Solanum tuberosum; Estrés oxidativo; Obesidad; Polifenoles (Fuente: DeCS BIREME).

\section{EFFECT OF Solanum tuberosum PEEL OR PULP ON ORGAN WEIGHT AND HEPATIC OXIDATIVE STRESS IN OBESE RATS}

\begin{abstract}
This study aimed to determine the effect of the consumption of peel flour or pulp flour from two varieties of Solanum tuberosum (Yungay potato and Canchán potato) on the accumulation of adipose tissue, organ weight, and oxidative stress in the liver of rats. We carried out an experimental study in 24 obese Holtzman rats, divided into four groups and subjected to diets containing 10\% flour from both varieties of potato. The groups were T1: Yungay peel; T2: Yungay pulp; T3: Canchán peel; and T4: Canchán pulp. When the study was completed, all the animals were slaughtered to record the weights of organs and adipose tissue and to extract samples to determine the enzyme activity of superoxide dismutase and catalase in the liver. The group of obese rats that consumed the pulp of the Yungay variety had less oxidative stress in the liver. Also, regardless of the tuber part consumed, this variety reduced the weight of the kidneys.
\end{abstract}

Keywords: Solanum tuberosum; Oxidative stress; Obesity; Polyphenols (Fuente: MeSH NLM).

\section{INTRODUCCIÓN}

Existe un incremento de enfermedades crónicas no transmisibles, tales como enfermedades cardiovasculares, algunos tipos de cáncer, hipertensión, dislipidemia, enfermedad crónica pulmonar y diabetes mellitus. Según la Organización Mundial de la Salud estas enfermedades causaron el $66 \%$ del total de muertes en Perú (1); asimismo, se afirma que el sobrepeso y la obesidad son factores que favorecen el desarrollo de estas enfermedades. Ante esta problemática existe una creciente tendencia por consumir alimentos funcionales debido a sus efectos beneficiosos para la salud; además de proteger a los tejidos del cuerpo contra el estrés oxidativo ${ }^{(2-4)}$.

Los antioxidantes difieren en su concentración según la parte del alimento en donde se encuentren. Por ejemplo, la papa es considerada un alimento funcional

Departamento de Nutrición, Universidad Nacional Agraria La Molina. Lima, Perú.

Ingeniero en Industrias Alimentarias, magíster Scientiae en Nutrición; ${ }^{\mathrm{b}}$ ingeniero en Industrias Alimentarias, Ph.D. en Nutrición; ${ }^{\mathrm{c}}$ médico veterinario, magíster Scientiae en Nutrición; ${ }^{d}$ nutricionista, Ph.D. en Nutrición

Recibido: 12/02/2019 Aprobado: 04/09/2019 En línea: 06/11/2019

Parte de los resultados del artículo fueron presentados como poster en el «International Congress of Nutrition» que se desarrolló en Argentina en octubre del 2017.

Citar como: Ortiz-Rojas VJ, Bernuy-Osorio ND, Zea-Mendoza OA, Vílchez-Perales C. Efecto de la cáscara o pulpa de Solanum tuberosum sobre el peso de órganos y estrés oxidativo hepático en ratas obesas. Rev Peru Med Exp Salud Publica. 2019;36(4):681-6. doi: 10.17843/rpmesp.2019.360.4238. 
cuyo poder antioxidante está dado por su contenido en polifenoles, vitamina $\mathrm{C}$ y carotenos ${ }^{(5)}$, la concentración de polifenoles es diez veces mayor en la cáscara que en la pulpa. Además, se afirma que el contenido de polifenoles difiere entre las diferentes variedades de papa, siendo las de pulpa coloreada las de mayor contenido en compuestos bioactivos ${ }^{(6)}$. Un estudio realizado en ratas ha demostrado que la administración de dietas que contienen cáscara de papa reduce el daño por estrés oxidativo y disminuye los niveles de glucosa en sangre (7); sin embargo, no existe evidencia del efecto del consumo de variedades de papa peruanas, considerando la gran diversidad de este tubérculo en Perú.

Por lo tanto, la presente investigación tiene como objetivo determinar el efecto del consumo de harina de cáscara o harina de pulpa de dos variedades de Solanum tuberosum (papa Yungay y papa Canchán) sobre la acumulación de tejido adiposo, peso de órganos y estrés oxidativo hepático en ratas obesas.

\section{EL ESTUDIO}

\section{DISEÑO DEL ESTUDIO}

La presente investigación tiene un diseño experimental y fue realizado siguiendo las directrices ARRIVE (Animal Research: Reporting of In Vivo Experiments).

\section{OBTENCIÓN DE HARINAS DE CÁSCARA Y DE PULPA DE DOS VARIEDADES DE PAPA}

Las variedades de papa, Canchán y Yungay, fueron adquiridas del mercado local; las cuales fueron llevadas a un agrónomo experto de la Universidad Nacional Agraria La Molina para su correspondiente identificación. Para la obtención de harina de cada variedad, éstas fueron lavadas manualmente hasta tres veces con agua corriente y posteriormente secadas. Por cada variedad, la cáscara fue extraída con ayuda de un pelador manual y la pulpa fue cortada en láminas de aproximadamente $0,5 \mathrm{~cm}$; ambas fueron sometidas a secado a $70^{\circ} \mathrm{C}$ por cinco horas (Memmert, Modelo UF55) según Rytel et al. ${ }^{(9)}$. Después, la cáscara y pulpa secas de las dos variedades fueron molidas (Figura 1). Finalmente, las harinas de cáscara y de pulpa de papa fueron incorporados en el $10 \%$ de la dieta obesogénica de las ratas (Figura 2) obteniendo los siguientes tratamientos T1: dieta con harina de cáscara Yungay, T2: dieta con harina de pulpa Yungay, T3: dieta con harina de cáscara Canchán y T4: dieta con harina de pulpa Canchán.

Investigaciones señalan que es posible inducir la obesidad mediante el consumo de dietas altas en grasa ${ }^{(10)}$, por lo que las dietas obesogénicas fueron elaboradas según los requerimientos del National Research Council (11), donde

\section{MENSAJES CLAVE}

Motivación para realizar el estudio. La obesidad no sólo produce acumulación excesiva de grasa sino también incrementa el daño oxidativo en el organismo. Una alternativa para mitigar esta enfermedad es a través del consumo de alimentos funcionales como la papa, que contiene compuestos bioactivos.

Principales hallazgos. El consumo de pulpa de la variedad Yungay disminuyó el estrés oxidativo en el hígado de ratas obesas causado por el consumo de una dieta alta en grasa. Además, la variedad Yungay independientemente de las partes del tubérculo redujo el peso de los riñones de las ratas obesas.

Implicancias. El consumo de harina de pulpa Yungay podría ser una alternativa accesible para favorecer la disminución del estrés oxidativo en el hígado de individuos obesos.

el nivel normal de energía metabolizable es de $4,1 \mathrm{kcal} / \mathrm{g}$, el mismo que fue superado en un $10 \%$ para las dietas obesogénicas $(4,41 \mathrm{kcal} / \mathrm{g})$, y fueron administradas de forma ad libitum (Tabla 1).

\section{POLIFENOLES TOTALES}

Se pesó cinco gramos de harina de pulpa y de cáscara de cada variedad, se sometió a extracción hidroalcohólica con etanol (20\% por 24 horas), después se centrifugó a 2500 rpm por cinco minutos (Kert Lab, Modelo 0412-1) considerando el sobrenandante para la cuantificación de polifenoles totales mediante el método de Singleton \& Rossi ${ }^{(12)}$.

\section{ANIMALES}

Se utilizaron 24 ratas Holtzman machos de dos meses y medio de edad, adquiridos del bioterio de la Universidad

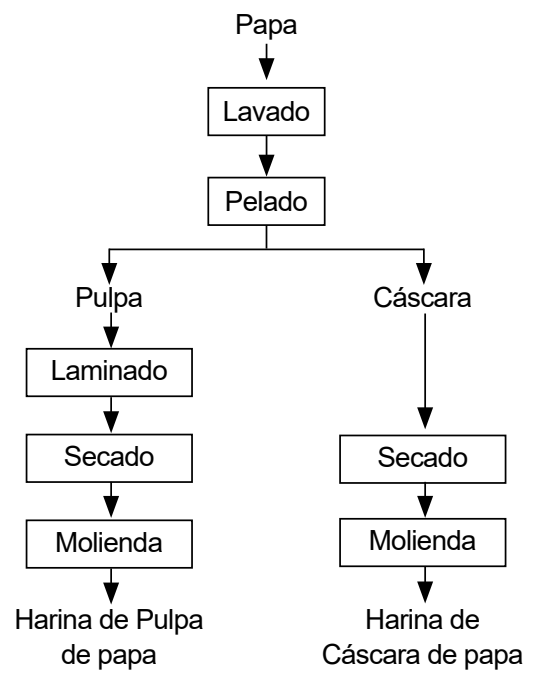

Figura 1. Flujograma de elaboración de harina de pulpa y harina de cáscara de dos variedades de Solanum tuberosum (papa Yungay y papa Canchán) 


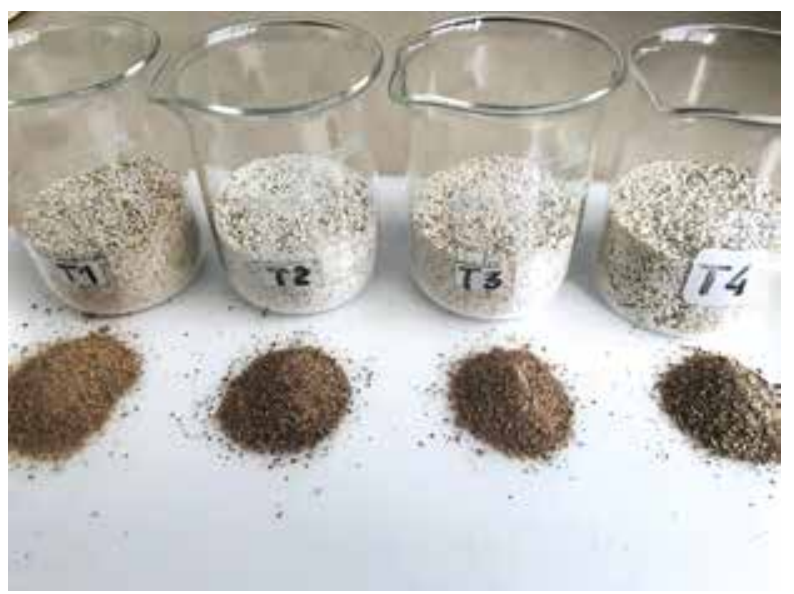

Figura 2. Dietas altas en grasa con $10 \%$ de harina de cáscara o pulpa de las dos variedades de papa (superior). Harina de cáscara y pulpa de dos variedades de papa (inferior). T1: dieta con harina de cáscara Yungay, T2: dieta con harina de pulpa Yungay, T3: dieta con harina de cáscara Canchán, T4: dieta con harina de pulpa Canchán

Nacional Agraria La Molina, que fueron alimentadas con dietas altas en grasas durante 55 días hasta cumplir los lineamientos somáticos de obesidad. Fueron colocados en jaulas individuales y distribuidos al azar en cuatro grupos, considerando seis ratas por grupo. Durante 21 días recibieron dietas altas en grasas con inclusión de harina de cáscara o harina de pulpa de las variedades de papa en estudio. Se registró el peso corporal y el consumo de alimento de forma diaria.

\section{PESADO DE ÓRGANOS Y TEJIDO ADIPOSO}

Los animales fueron sacrificados mediante punción cardiaca, este procedimiento fue realizado por un médico veterinario siguiendo las normas éticas para animales de laboratorio. Luego de verificar la muerte clínica de las ratas, se procedió a hacer un corte en el abdomen para extraer los órganos: hígado, riñones, intestino delgado y tejido adiposo considerando grasa subcutánea abdominal y grasa visceral (suma de grasa gonadal, mesentérica y retroperitoneal). Conforme se iban extrayendo los órganos y tejidos se procedió a lavarlos con suero fisiológico para finalmente secarlos con papel absorbente y registrar el peso.

\section{SISTEMA ENZIMÁTICO HEPÁTICO}

Se utilizó un gramo de hígado previamente lavado con $\mathrm{KCl}$ $(0,154 \mathrm{M})$ y buffer fosfato salino (PBS) $\mathrm{pH} 7,4$. La muestra fue homogenizada con buffer PBS al $10 \%$, después se centrifugó a $2500 \mathrm{rpm}$ por cinco minutos a cuatro grados centígrados (MPW 380-R) para finalmente extraer el sobrenadante para las determinaciones de superóxido dismutasa (SOD), Marklund \& Marklund (13) y catalasa (CAT) Aebi ${ }^{(14)}$. Los resultados fueron expresados como actividad específica de la enzima en el hepatocito a través del método de Lowry, et al. ${ }^{(15)}$, donde una unidad de actividad SOD es la cantidad de muestra necesaria para alcanzar el $50 \%$ de inhibición (USOD/mg) y una unidad de catalasa $(\mathrm{mU} / \mathrm{mg})$ equivale a la cantidad de enzima que cataliza la descomposición de un micromol de peróxido de hidrógeno por minuto.

\section{ANÁLISIS ESTADÍSTICO}

Los pesos del tejido adiposo y de los órganos, y el sistema enzimático hepático fueron analizados mediante la prueba ANOVA. Para determinar si existen diferencias entre los tratamientos se utilizó la prueba de Tukey ${ }^{(16)}$ mediante el paquete estadístico Minitab v.17.1.0. Los valores de $p<0,05$ fueron considerados como estadísticamente significativos.

\section{CONSIDERACIONES ÉTICAS}

Todos los animales de experimentación fueron tratados según los lineamientos del Centro Interdisciplinario de

Tabla 1. Composición y análisis proximal de dietas experimentales que contienen $10 \%$ de harina de pulpa o cáscara de dos variedades de Solanum tuberosum (papa Yungay y papa Canchán)

\begin{tabular}{|c|c|c|c|c|}
\hline \multirow{2}{*}{ Ingredientes } & \multicolumn{4}{|c|}{ Tratamientos \% } \\
\hline & T1 & T2 & T3 & T4 \\
\hline \multicolumn{5}{|l|}{ Composición } \\
\hline Almidón de maíz a & 52,9 & 51,8 & 52,9 & 51,5 \\
\hline Manteca vegetal & 19,9 & 19,9 & 19,7 & 20,0 \\
\hline Caseína $^{\mathrm{b}}$ & 13,6 & 14,0 & 13,5 & 14,0 \\
\hline Minerales ${ }^{c}$ & 1,4 & 1,7 & 1,6 & 1,7 \\
\hline Vitaminas $^{d}$ & 1,0 & 1,0 & 1,0 & 1,0 \\
\hline Fibra $^{e}$ & 1,2 & 1,7 & 1,3 & 1,8 \\
\hline Insumo evaluado & 10,0 & 10,0 & 10,0 & 10,0 \\
\hline Total & 100,0 & 100,0 & 100,0 & 100,0 \\
\hline Energía metabolizable (kcal/g) & 4,41 & 4,41 & 4,41 & 4,41 \\
\hline \multicolumn{5}{|c|}{ Análisis proximal determinado } \\
\hline Proteína $^{f}$ & 13,7 & 15,3 & 15,1 & 15,6 \\
\hline Grasa $^{9}$ & 20,5 & 20,1 & 20,9 & 20,9 \\
\hline Fibra cruda ${ }^{h}$ & 2,5 & 1,6 & 2,5 & 1,5 \\
\hline Ceniza $^{i}$ & 2,4 & 2,2 & 2,0 & 2,2 \\
\hline ELN $^{j}$ & 60,9 & 60,8 & 59,5 & 59,8 \\
\hline
\end{tabular}

T1: cáscara de papa Yungay, T2: pulpa de papa Yungay, T3: cáscara de papa Canchán, T4: pulpa de papa Canchán

a Almidón de maíz MP Biomedicals.

b Caseína MP Biomedicals: proteína $\geq 95,0 \%$

c Mix de minerales MP Biomedicals (AIN-76)

d Mix de vitaminas MP Biomedicals (AIN-76)

e Fibra a-celulosa MP Biomedicals: celulosa 99\%, contenido de fibra $99,9 \%$

f Proteína total $(\mathrm{N} \times 6.25)$, AOAC (2005) 984.13

g Grasa, ANKOM Technology Method (AOCS Official Procedure Am 5-04)

h Fibra cruda ANKOM Filter Bag Technique (AOCS Approved

Procedure $\mathrm{Ba}$ 6a-05)

Ceniza AOAC (2005) 942.05

Extracto libre de nitrógeno (ELN) 
Tabla 2. Extracción de órganos, de tejido adiposo y sistema enzimático hepático de ratas obesas alimentadas con dietas conteniendo cáscara o pulpa de dos variedades de papa.

\begin{tabular}{|c|c|c|c|c|c|c|c|}
\hline \multirow[b]{2}{*}{ Dieta } & \multicolumn{3}{|c|}{ Peso de órganos (g) } & \multicolumn{2}{|c|}{ Peso de tejido adiposo (g) } & \multicolumn{2}{|c|}{ Sistema enzimático hepático } \\
\hline & Hígado & $\begin{array}{l}\text { Intestino } \\
\text { delgado }\end{array}$ & Riñones & Abdominal & Visceral & $\begin{array}{c}\text { SOD } \\
\text { (USOD/mg) }\end{array}$ & $\begin{array}{c}\text { CAT } \\
(\mathrm{mU} / \mathrm{mg})\end{array}$ \\
\hline \multicolumn{8}{|l|}{ Variedad } \\
\hline Yungay & $13,3(1,6)$ & $9,9(1,2)$ & $2,6(0,3)$ & $5,6(1,4)$ & $16,7(2,8)$ & $41,7(7,9)$ & $139,5(21,3)$ \\
\hline Canchán & $13,9(1,6)$ & $9,9(1,6)$ & $2,9(0,5)$ & $5,9(1,5)$ & $16,5(4,8)$ & $40,4(3,9)$ & $120,1(14,2)$ \\
\hline Valor de $p$ & 0,374 & 0,998 & 0,032 & 0,571 & 0,925 & 0,451 & 0,008 \\
\hline \multicolumn{8}{|l|}{ Parte del tubérculo } \\
\hline Cáscara & $13,2(1,8)$ & $10,2(1,5)$ & $2,7(0,4)$ & $5,8(1,5)$ & $16,0(3,1)$ & $40,1(4,6)$ & $126,4(18,3)$ \\
\hline Pulpa & $13,9(1,4)$ & $9,7(1,2)$ & $2,9(0,4)$ & $5,6(1,3)$ & $17,2(4,5)$ & $42,0(7,4)$ & $133,1(22,3)$ \\
\hline Valor de $p$ & 0,339 & 0,376 & 0,146 & 0,799 & 0,433 & 0,890 & 0,320 \\
\hline \multicolumn{8}{|l|}{ Interacción } \\
\hline Yungay Cáscara & $13,2^{a}(2,0)$ & $10,3^{a}(0,8)$ & $2,5^{a}(0,2)$ & $5,8^{a}(1,2)$ & $16,7^{\text {a }}(2,4)$ & $38,6^{b}(4,5)$ & $127,5^{\mathrm{a}}(20,2)$ \\
\hline Yungay Pulpa & $13,4^{\mathrm{a}}(1,3)$ & $9,5^{\mathrm{a}}(1,4)$ & $2,6^{a}(0,3)$ & $5,4^{\mathrm{a}}(1,6)$ & $16,6^{\mathrm{a}}(3,3)$ & $44,8^{a}(3,6)$ & $151,4^{\mathrm{a}}(15,7)$ \\
\hline Canchán Cáscara & $13,3^{\mathrm{a}}(1,7)$ & $10,1^{\mathrm{a}}(2,0)$ & $2,8^{a}(0,5)$ & $5,9^{a}(1,9)$ & $15,2^{\mathrm{a}}(3,8)$ & $41,6^{\mathrm{a}}(4,6)$ & $125,4^{\mathrm{b}}(18,0)$ \\
\hline Canchán Pulpa & $14,4^{\mathrm{a}}(1,5)$ & $9,8^{\mathrm{a}}(0,9)$ & $3,1^{\mathrm{a}}(0,4)$ & $6,0^{\mathrm{a}}(0,9)$ & $17,9^{\mathrm{a}}(5,6)$ & $39,2^{b}(3,3)$ & $114,9^{b}(7,2)$ \\
\hline Valor de $p$ & 0,498 & 0,656 & 0,443 & 0,681 & 0,406 & 0,025 & 0,017 \\
\hline
\end{tabular}

Los resultados se presentan como media por grupo y su desviación estándar entre paréntesis

SOD: superóxido dismutasa, CAT: catalasa

$a-b$ : diferentes superíndices dentro de una columna indica diferencias $(p<0,05)$

Estudios en Bioética de la Universidad de Chile para el cuidado y uso de animales de laboratorio ${ }^{(8)}$.

\section{HALLAZGOS}

\section{POLIFENOLES TOTALES}

El contenido de polifenoles totales fue mayor en la harina de cáscara de la variedad Yungay $(7,78 \mathrm{mg}$ ác. clorogénico eq./g) seguido por la harina de cáscara de la variedad Canchán (6,49 mg ác. clorogénico eq./g), y éstas a su vez fueron superiores a los obtenidos de la pulpa de ambas variedades (pulpa Yungay 2,46 mg ác. clorogénico eq./g; pulpa Canchán 1,03 mg ác. clorogénico eq./g).

\section{EXTRACCIÓN DE ÓRGANOS Y TEJIDO ADIPOSO}

Se obtuvo diferencias significativas entre variedades $(p<0,05)$ para el peso de los riñones a favor de la variedad Yungay. No se encontraron diferencias para el peso del hígado, intestino delgado, tejido adiposo abdominal y visceral entre los grupos experimentales (Tabla 2).

\section{SISTEMA ENZIMÁTICO HEPÁTICO}

A pesar de que no hubo diferencias entre la variedad y parte de tubérculo para la actividad de la enzima SOD ( $p>0,05)$, los mayores promedios corresponden a la variedad Yungay y pulpa, respectivamente; lo que promueve una interacción significativa para pulpa de la variedad Yungay $(p<0,05)$, con un mayor valor de su promedio. Asimismo, sólo se encontró diferencias en la variedad para la enzima CAT $(p<0,05)$ con mayor valor para la variedad Yungay (Tabla 2).

\section{DISCUSIÓN}

Los valores de polifenoles totales obtenidos superan a lo reportado por Singh et al ${ }^{(7)}$, quienes alimentaron con harina de cáscara de papa (3,9 mg ác. clorogénico eq./g) a ratas diabéticas inducidas con estreptozotocina. Existe escasa evidencia científica sobre el contenido de polifenoles totales en cáscara o pulpa de papa según su variedad. Sin embargo, se puede indicar que el contenido de estos compuestos presentes en la cáscara de la variedad Yungay y Canchán son tan elevados como los blueberries $(6,70 \mathrm{mg}$ ác. clorogénico eq./g) y fresas (3,63 mg ác. clorogénico eq./g), que son frutos que se caracterizan por tener un alto contenido fenólico ${ }^{(17)}$.

Respecto al peso de órganos, Yoshino et al (18) obtuvieron un similar peso promedio para los riñones $(2,11 \pm 0,55 \mathrm{~g})$ de ratas obesas de semejante edad que fueron suplementadas con $1 \%$ de polifenoles extraídos de hojas de té. Asimismo, Piao et al ${ }^{(19)}$ en su investigación sobre el cambio en el peso de órganos de ratas sanas a diferentes edades determinaron que el peso de los riñones en ratas de similar edad a las utilizadas en el presente experimento fue de $3,30 \pm 0,67 \mathrm{~g}$, encontrándose los resultados obtenidos en ese rango.

Singh et al (7) reportaron un peso de hígado superior $(27,3 \pm 0,2 \mathrm{~g})$ a lo obtenido en el presente experimento 
debido a que se trató de ratas diabéticas inducidas por estreptozotocina, suplementadas con dieta al $10 \%$ de cáscara de papa. En otra investigación, el peso final de hígado de ratas sanas delgadas fue de $12,9 \pm 2,4 \mathrm{~g}$ mientras que los otros grupos de evaluación, control obesas y obesas suplementadas con extracto de ciruela, el peso llegó hasta $26,8 \pm 2,1$ y $21,5 \pm 1,4 \mathrm{~g}$; respectivamente ${ }^{(20)}$; por tanto, se puede indicar que el consumo de dietas conteniendo harina de papa permite lograr un peso del hígado muy cercano a aquellos animales en condición normal o sana. Asimismo, Piao et al ${ }^{(19)}$ obtuvieron un peso de hígado de ratas sanas de 11,24 $\pm 2,34 \mathrm{~g}$, hallándose los resultados obtenidos dentro de los pesos normales. Es necesario indicar que el consumo de dietas altas en grasa por un tiempo prolongado afecta el hígado debido al incremento de triglicéridos, afectando adversamente a todos los sistemas del cuerpo ${ }^{(20)}$.

Nakazato et al (21) reportaron la misma tendencia para la acumulación de tejido adiposo visceral con valores de $14,8 \pm 0,9 \mathrm{~g}$ en ratas obesas de edad similar suplementadas con $5 \%$ de polifenoles de manzana. Los investigadores sugieren que el aumento de la grasa visceral está relacionado con el incremento sistémico del estrés oxidativo en varios órganos involucrados en la patogénesis del síndrome metabólico.

A nivel de las enzimas hepáticas, Singh et al ${ }^{(7)}$ reportaron valores menores de SOD, pero mayores de CAT para ratas sanas $(16,7 \pm 2,0 \mathrm{USOD} / \mathrm{mg} ; 293,0 \pm 14,7 \mathrm{mU} / \mathrm{mg})$, diabéticas (18,8 \pm 2,6 USOD/mg; 238,8 $\pm 12,6 \mathrm{mU} / \mathrm{mg})$ y aquellas ratas diabéticas tratadas con $10 \%$ de harina de cáscara de papa (17,7 $\pm 3,0$ USOD/mg; $324,0 \pm 22,8 \mathrm{mU} / \mathrm{mg})$ en comparación a lo obtenido en el presente experimento. La acción de la enzima SOD tiene como finalidad lograr un $50 \%$ de inhibición de compuestos oxidantes y es la responsable de transformar el radical superóxido en $\mathrm{O}_{2} \mathrm{y}$ $\mathrm{H}_{2} \mathrm{O}_{2}$, para que posteriormente la CAT continúe su acción sobre el peróxido de hidrogeno convirtiéndolo en $\mathrm{O}_{2} \mathrm{y}$ $\mathrm{H}_{2} \mathrm{O}$, logrando así obtener productos inocuos para nuestro organismo ${ }^{(20)}$; es decir, en relación a nuestro experimento la mayor acción de SOD producto del consumo de las dietas produjo menor acción de la enzima CAT reflejándose en los valores obtenidos. Además, se ha demostrado que la capacidad antioxidante de los órganos en las ratas generalmente se mantiene sin grandes variaciones durante el envejecimiento ${ }^{(22)}$.

Hsieh et al (22) evaluaron la acción enzimática de SOD y CAT en el hígado de ratas alimentadas con dieta alta en colesterol y suplementada con extractos de cáscara de papa a diferentes concentraciones (1\%, 2\% y $3 \%$ ) concluyendo que se promueve un efecto hepatoprotector aminorando el estrés oxidativo, observándose una mejor respuesta a una mayor concentración. Este hallazgo concuerda con lo obtenido, a pesar de que el autor utilizó extractos de cáscara de papa a diferentes concentraciones, mientras que, en nuestro estudio las variedades de papa fueron suministradas de forma completa y directa en la dieta (cáscara o pulpa).

Entre las limitaciones del presente estudio, no se realizó la determinación de un tamaño de muestra, no se realizó la determinación de otros biomarcadores de estrés oxidativo, y además que basados en el modelo estadístico planteado no se tuvo un grupo control de forma paralela al experimento, lo que sugiere el desarrollo de nuevos estudios considerando un grupo control positivo (ratas obesas alimentadas con dieta alta en grasa) y un grupo control negativo (ratas sanas alimentadas con dieta estándar), para compararlas con las obesas que consumieron dietas a base de cáscara y pulpa de papa. A pesar de no haberse considerado un grupo control los resultados obtenidos muestran diferencias en el estrés oxidativo hepático entre los grupos que consumieron partes del tubérculo y variedades de papa, evidenciando una mejor respuesta en ratas en condición de obesidad.

Por tanto, se concluye que el consumo de harina de cáscara o pulpa de papa de las variedades estudiadas no afectó el peso del hígado e intestino delgado, ni la acumulación de tejido adiposo visceral y abdominal en ratas obesas. No obstante, el consumo de la variedad Yungay redujo el peso de los riñones en ratas obesas. Además, se obtuvo menor estrés oxidativo en el hígado del grupo que consumió la dieta con harina de pulpa Yungay, disminuyendo el daño ocasionado por los radicales libres.

Contribuciones de autoría: VJOR y CVP participaron en la concepción y diseño del artículo, recolección de datos y redacción de artículo. VJOR y NDBO participaron en análisis e interpretación de los datos. NDBO, OAZM y CVP realizaron la asesoría técnica y administrativa. Todos los autores aprobaron la versión final del artículo.

Fuentes de financiamiento: la investigación fue financiada por el Fondo Nacional de Desarrollo Científico y Tecnológico del Consejo Nacional de Ciencia, Tecnología e Innovación Tecnológica (FONDECYT-CONCYTEC) bajo el Convenio de Subvención $\mathrm{N}^{\circ}$ 275-2015-FONDECYT-PERÚ.

Conflictos de interés: los autores de la presente comunicación declaran que no tienen ningún conflicto de interés.

\section{REFERENCIAS BIBLIOGRÁFICAS}

1. Organización Mundial de la Salud. Enfermedades No Transmisibles. Nota descriptiva, Enerm 2015 [Internet]. Ginebra:OMS;2015 [citado el 24 de diciembre del 2018]. Disponible en: http://www.who.int/mediacentre/ factsheets/fs355/es/

2. Zhang H, Tsao R. Dietary polyphenols, oxidative stress and antioxidant and antiinflammatory effects. Curr Opin Food
Sci. 2016; 8:33-42. Doi: 10.1016/j. cofs.2016.02.002.

3. Rodriguez-Ramiro I, Vauzour D, Minihane AM. Polyphenols and non-alcoholic fatty 
liver disease: impact and mechanisms. Proc Nutr Soc. 2016;75(1):47-60. doi: $10.1017 /$ S0029665115004218.

4. Bahadoran Z, Mirmiran P, Azizi F. Dietary polyphenols as potential nutraceuticals in management of diabetes: a review. J Diabetes Metab Disord. 2013;12(1):43. doi: 10.1186/2251-6581-12-43.

5. McGill CR, Kurilich AC, Davignon J. The role of potatoes and potato components in cardiometabolic health: A review. Ann Med. 2013;45(7):467-73. doi: 10.3109/07853890.2013.813633.

6. Schieber A, Saldaña MD. Potato Peels: A Source of nutritionally and pharmacologically interesting compounds-A Review. Food. 2009;3(2):23-9.

7. Singh N, Kamath V, Rajini PS. Protective Effect of Potato Peel Powder in Ameliorating Oxidative Stress in Streptozotocin Diabetic Rats. Plant Foods Hum Nutr. 2005;60(2):49-54.

8. Cardozo CA, Mrad A, Martínez C, Rodríguez E, Lolas F. El animal como sujeto experimental: Aspectos técnicos y éticos [Internet]. Santiago: Centro Interdisciplinario de Estudios en Bioética de la Universidad de Chile; 2007 [citado el 18 de noviembre de 2018]. Disponible en: https://www.healthresearchweb.org/files/ libroanimales.pdf

9. Rytel E, Tajner-Czopek A, Kita A, Aniołowska M, Kucharska AZ, SokótŁętowska A, et al. Content of polyphenols in coloured and yellow fleshed potatoes during dices processing. Food Chem. 2014;161:224-9. doi: 10.1016/j. foodchem.2014.04.002.
10. Nascimento AF, Sugizaki MM, Leopoldo AS, Lima-Leopoldo AP, Luvizotto RAM, Nogueira CR, et al. A Hypercaloric pelletdiet cycle induces obesity and comorbidities in wistar rats. Arq Bras Endocrinol Metabol. 2008;52(6):968-74.

11. National Research Council. Nutrient Requirements of Laboratory Animals [Internet]. Washington DC: National Academy Press; 1995 [citado 3 septiembre de 2018]. Disponible en: https://www. ncbi.nlm.nih.gov/books/NBK231927

12. Singleton VL, Rossi JA. Colorimetry of Total Phenolics with PhosphomolybdicPhosphotungstic Acid Reagents. Am J Enol Vitic. 1965;16:144-58.

13. Marklund S, Marklund G. Involvement of the superoxide anion radical in the autoxidation of pyrogallol and a convenient assay for superoxide dismutase. Eur J Biochem. 1974; 47(3):469-74.

14. Aebi H. Catalase in vitro. Methods Enzymol. 1984;105:121-6. Doi: 10.1016/ S0076-6879(84)05016-3.

15. Lowry $\mathrm{OH}$, Rosebrough NJ, Farr AL, Randall RJ. Protein measurement with the Folin phenol reagent. J Biol Chem. 1951;193(1):265-75.

16. Kuehl R. Diseño de experimentos: Principios estadísticos para el diseño y análisis de investigaciones. $2^{\mathrm{a}}$ ed. México: Thomson Editores; 2011.

17. Cho KD, Han CK, Lee BH. Loss of Body Weight and Fat and Improved Lipid Profiles in Obese Rats Fed Apple Pomace or Apple Juice Concentrate. J Med Food. 2013;16(9):823-30. doi: 10.1089/ jmf.2013.2784.
18. Yoshino K, Tomita I, Sano M, Oguni I, Hara Y, Nakano M. Effects on Long-Term dietary supplement of tea polyphenols on lipid peroxide levels in rats. Age. 1994;17(3):79-85.

19. Piao Y, Liu Y, Xie X. Change Trends of Organ Weight Background Data in Sprague Dawley Rats at Different Ages. J Toxicol Pathol. 2013;26(1):29-34. doi: 10.1293/tox.26.29.

20. Noratto G, Martino H, Simbo S, Byrne $D$, Mertens-Talcott S. Consumption of polyphenol-rich peach and plum juice prevents risks factors for obesity-related metabolic disorders and cardiovascular Disease in Zucker rats. J Nutr Biochem. 2015;26(6):633-41. doi: 10.1016/j. jnutbio.2014.12.014.

21. Nakazato K, Song H, Waga T. Effects of Dietary Apple Polyphenol on Adipose Tissues Weights in Wistar Rats. Exp Anim. 2006;55(4):383-9. doi: 10.1538/ expanim.55.383.

22. Hsieh YL, Yeh YH, Lee YT, Huang CY. Dietary potato peel extract reduces the toxicity of cholesterol oxidation products in rats. J Funct Foods. 2016;27:461-71. doi: 10.1016/j.jff.2016.09.019.

Correspondencia: Viviana J. Ortiz Rojas Dirección: Calle Nevado Ausangate 185. Urb. Aprovisa, La Molina. Lima, Perú Teléfono: (+51) 958834635

Correo electrónico:viv.mwg@gmail.com 